\title{
Variations de la perte de charge du canal d'amenée de la centrale de Bévercé
}

\author{
Variation of the head losses \\ in the Bévercé plant head-race tunnel
}

P'AR

ALr. SCHLAG,

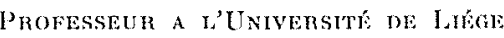

rit

\author{
R. STMONS
}

INGÉNIEU A LA SOFINA

English synopsis, p. 577.

La centrale de Bévercé, appartenant au groupement des «Centrales Electriques de l'EntreSambre-et-Meuse et de la région de Malmedy », filiale de la Sofina, est située en Belgique, à proximité de la frontière allemande; elle est alimentée par le réservoir artificiel de Robertville, cré par un harrage établi sur la Warche (fig. 1).

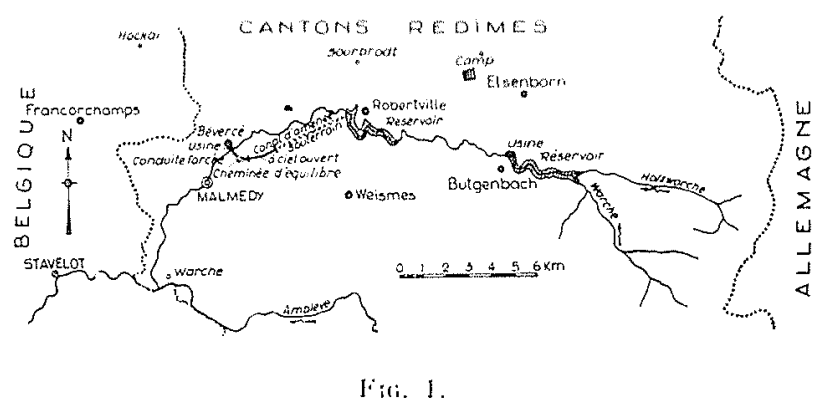

L'eau est retenue à la cote normale de $496 \mathrm{~m}$. Du réservoir part un canal d'amenée sous pression, dont la partie amont, comportant environ les $4 / 5$ de la longueur, est constituée par un tunnel, tandis que dans le tronçon aval, sur le dernier cinquième, le tunnel est remplacé par une tuyauterie métallique rivée, courant à flanc de coteau.

La conduite d'amenée, d'une longueur totale de $5.465 \mathrm{~m}$, peut être divisée en cinq parties, composées chacune de tronçons non consécutifs, mais pouvant se grouper comme suit, d'après leur nature :

\begin{tabular}{|c|c|c|}
\hline & $\begin{array}{l}\text { Longuent } \\
(\mathrm{m})\end{array}$ & $\begin{array}{c}\text { Section } \\
\left(\mathrm{m}^{2}\right)\end{array}$ \\
\hline (a) Béton lisse.. & 1.252 & 2,68 \\
\hline (b) Béton lisse.......... & 2.600 & 2,98 \\
\hline $\begin{array}{c}\text { c) Fond lisse, parois gunite, } \\
\text { irrégulières } \ldots \ldots \ldots\end{array}$ & 420 & 3,80 \\
\hline $\begin{array}{l}\text { (d) Fond lisse, parois gunite, } \\
\text { lissées a la truelle.... }\end{array}$ & 120 & 3,80 \\
\hline TOTAL pOUn $a$ à $a \ldots$ & 4.398 & \\
\hline $\begin{array}{l}\text { e) Conduite métallique ou } \\
\text { tunnel recouvert de mé- } \\
\text { tal ................ }\end{array}$ & 1.067 & 3,10 \\
\hline & 5.465 & \\
\hline
\end{tabular}

La section moyenne, compte tenu des longueurs, est, pour l'ensemble des parties $a \dot{a} d$, exactement de $3 \mathrm{~m}^{2}$ correspondant à un diamètre de $1,955 \mathrm{~m}$; pour la conduite d'amenée entière, elle est de $3,02 \mathrm{~m}^{2}$, correspondant à un diamètre de $1,96 \mathrm{~m}$.

Nous admettrons dans les deux cas une valeur de $3 \mathrm{~m}^{2}$.

La conduite d'amenée aboutit à une cheminéc d'écuilibre d'où part la conduite forcée, en tôle d'acier rivée, longue de $265 \mathrm{~m}$ et d'un diamètre intérieur de 1,755 m. Elle se termine à la centrale de Bévercé. La hauteur de chute est de $154 \mathrm{~m}$. La centrale comporte trois groupes de $5.700 \mathrm{ch}$, soit une puissance totale installée de 
$11.500 \mathrm{~kW}$ environ (Lurbines Escher-Wyss du type Francis à axe horizontal, tommant à $750 \mathrm{t} / \mathrm{m}$, alternateurs SEM de 5.500 kiVA à $6 \mathrm{kV}$ ).

Des essais furent exécutés sur l'installation les 10 juin 1932 et 30 juillet 1935 , dans le but de fixer le coefficient de conversion, c'est-à-dire le nombre de watts-heure fourni par $\mathrm{m}^{3}$ d'eau turbinée, et accessoirement de contròler le rendement des machines et les pertes de charge dans les ouvrages d'amenée d'eau.

En 1947, il fut estimé nécessaire d'entreprendre de nouveaux essais; on avait en effet constaté une réduction importante de la puissance maximum productible (qui était tombée de $10.100 \mathrm{~kW}$ en 1930 à $8.600 \mathrm{~kW}$ en 1947 ). Elle fut attribuée à un accroissement de la perte de charge dans la conduite d'amenée, en raison des deux constatations suivantes :

Une machine débitant seule continuait à produire pratiquement la mème puissance qu'autrefois;

Les trois machines démarrées rapidement continuaient à donner une puissance voisine de $10.000 \mathrm{~kW}$ pendant un court moment (alimentation par la cheminée d'équilibre).

De nouveaux essais effectućs le 12 avril 1949 confirmèrent cette supposition : le coefficient de résistance $\lambda$ moyen de la conduite d'amenée a été trouvé égal à 0,0263 contre 0,0202 aux essais de 1932 et 0,0208 à ceux de 1935 , soil une augmentation de 30 ou de $26,5 \%$.

Il fut décidé en conséquence de procéder au netloyage de la conduite d'amenée. Cette opération eut lieu en septembre-octobre 1949. Elle fut limitée, faute de temps, à la seule partie en tunnel de la conduite d'amenée, la partie métallique ayant d'ailleurs déjà été nettoyéc en 193537 en vue de la repeindre.

On constata dans la conduite d'amenée un dépôt de boues de couleur noire et d'épaisseur assez uniforme, comprise entre 12 et $17 \mathrm{~mm}$; sa surface extérieure présentait des ondulations de même nature que celles qu'ofrre le sable des plages. Les dimensions de ces ondulations ne furent malheureusement pas relevées; les ouvriers qui avaient procédé au nettoyage ont été interrogés par la suite; leurs estimations variaient dans de très fortes proportions. Il semble (mais ces valeurs doivent être acceptées avec prudence) que la hauteur des ondulations variait de 2 à $6 \mathrm{~mm}$, avec une moyenne de $3,5 \mathrm{~mm}$, tandis que l'écart de deux ondulations successives avait été apprécié comme variant de 20 à $500 \mathrm{~mm}$, avec une moyenne de l'ordre de $110 \mathrm{~mm}$.

Aussitôt après la vidange de la conduite, la consistance de ce dépôt était molle et le nettoyage pouvait se faire à la main. Il se produisait ensuite un durcissement à l'air, et des raclettes en tòle ont dû être employées.
Un échantillon prélevé à une hauteur de $1,40 \mathrm{~m}$ de la conduite a cté analysé par les Laboratoires de la Sofina. L'analyse a donné les résultats suivants :

\section{Composition GÉNERALE:}

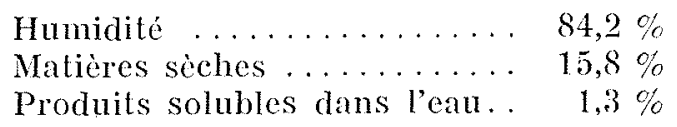

COMposition Du produte sec :

Pertes au feu............

Manganèse (caleulé en bi-oxyde de $\mathrm{Mn}) \ldots \ldots \ldots \ldots \ldots \ldots$

Silice $\ldots \ldots \ldots \ldots \ldots \ldots \ldots$

Oxyde de fer.............

Alumine $\ldots \ldots \ldots \ldots \ldots \ldots$.

Oxyde de zinc............

Oxyde de baryum..........

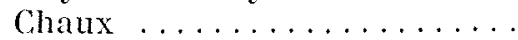

$16,4 \%$
$57,6 \%$
$16,4 \%$
$7,6 \%$
$5,3 \%$
$0,9 \%$
$0,9 \%$
$5,6 \%$

Absence de magnésie, anhydride sulfurique, chlore, anhydride phosphorique.

Après le nettoyage, de nouvelles mesures de perte de charge furent effectuées le 30 novembre 1949. Elles donnèrent pour l'ensemble de la conduite d'amenée un coefficient de résistance $\lambda$ de 0,0154 , soit inférieur de $24 \%$ à la valeur 1932 et de $41,5 \%$ à la valeur 1949 avant netloyage. D'autre part, dès le 10 octobre 1949, une puissance de 9.400 à $9.500 \mathrm{~kW}$ put être obtenue pour une cote de 490,70 au lac de Robertville (contre $8.600 \mathrm{~kW}$ avant nettoyage pour une cote de 495).

Organisation des ressais. - Les essais devaient comporter la mesure des débits et celle des différences de charges entre les deux extrémités du canal d'amenée.

Le débit a été déterminé dans une section de la conduite forcée, par la méthode du moulinet. Les vitesses ont été relevées en 9 points d'un diamètre vertical et le débit en a été déduit par intégration graphique du diagramme des vitesses. La section de mesure étant située à une distance de 34 diamètres du coude amont le plus proche, il a été admis qu'il suffisait de faire le relevé des vitesses selon un seul diamètre.

Lors des essais du 12 avril 1949 est apparue une petite incertitude quant à l'aire exacte de la section de mesure : alors que le diametre intérieur vertical mesuré était de $1,7555 \mathrm{~m}$, le périmètre extérieur mesuré de la virole était de $5,504 \mathrm{~m}$ correspondant, compte tenu de l'épaisseur, à un diamètre intérieur de $1,752 \mathrm{~m}$. Sa section semble done s'être déformée et si l'on admet qu'elle est devenue elliptique, on trouve que sa surface est de $4 \%$ inféricure à celle d'un cercle de $1,7555 \mathrm{~m}$ de diamètre. Pour rendre les résultats de 1949 comparables à ceux de 1932 
et 1935, on a toutefois admis que la section élait circulaire el de $1,7555 \mathrm{~m}$ de diametre. Il se pourrait donc que les débits calculés en 1949 soient surestimés d'environ $4 \%$.

La perte de charge dans le canal d'amence résulte de la différence des niveaux d'eau dans le réservoir de Robertville et dans la cheminée d'écquilibre.

Le niveau d'amont a été relevé avec soin à l'échelle limnimétrique du lac d'alimentation.

Le niveau dans la chemince d'équilibre a été mesuré à l'aide d'un dispositif, constitué d'un flotteur suspendu à un câble passant sur 2 poulies et équilibré par un contrepoids. Un repire lixé au câble permetlait de suivre les déplacements du flotteur : sa position «zéro » avait dé préalablement déterminée, lorsque, aucun débil ne traversant le canal d'amenée, le niveau dams la chemince diat illenlique an niveau du line.

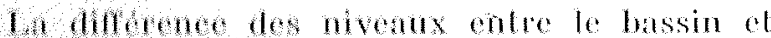
to chemine dequilibre "ste corvige, pour donnei li perte de charge:

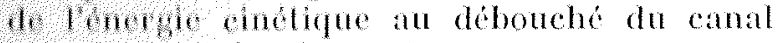
Il homor dans la cheminée,

4. pout certains essais, de la perte (calculée) propre it un pelit débil $\left(+0,035 \mathrm{~m}^{3} / \mathrm{s}\right.$ ) alimentant tmo station d'ozonisation, installée à environ $700 \mathrm{~m}$ en amont de la cheminéc.
Pour la plupart des essais, cette station avait d'ailleurs été arrètée.

INTERPRÉTATION DES RÉSULTATS DES ESSAIS. De la connaissance du débit $Q$ et de la perte de charge totale $H$ dans le canal d'amence, il était aisé de déterminer le coefficient de résistance $x$ et lo nombre de Reynolds, par les formules:

$$
\mathrm{H}=\frac{\lambda}{\mathrm{D}} \cdot \frac{\mathrm{U}^{2}}{2 g} \cdot \quad \mathrm{L} \text { et } \mathrm{Re}=\frac{\mathrm{UI})}{\nu}
$$

(U $=$ vitesse moyenne).

On a arbitrairement adopté pour y les valeurs correspondant aux températures suivantes (les tempéralures de l'eau n'ayant pas été relevées):

$12^{\circ}$ pour les essais du 10 juin 1932 ,

$15^{\circ}$ pour les essais du 30 juillet 1935 ,

$5^{\circ}$ pour les essais du 12 avril 1949 ,

2 ' pour les essais du 30 novembre 1949

La connaissance exacte de la température ne parait pas avoir d'ailleurs une importance essenticlle.

Nous avons pu de la sorte reporter les résultats obtenus sur le diagramme elassique de Nrkuradse (fig. 2).

On voit que les valeurs moyennes de $\lambda$ peu-

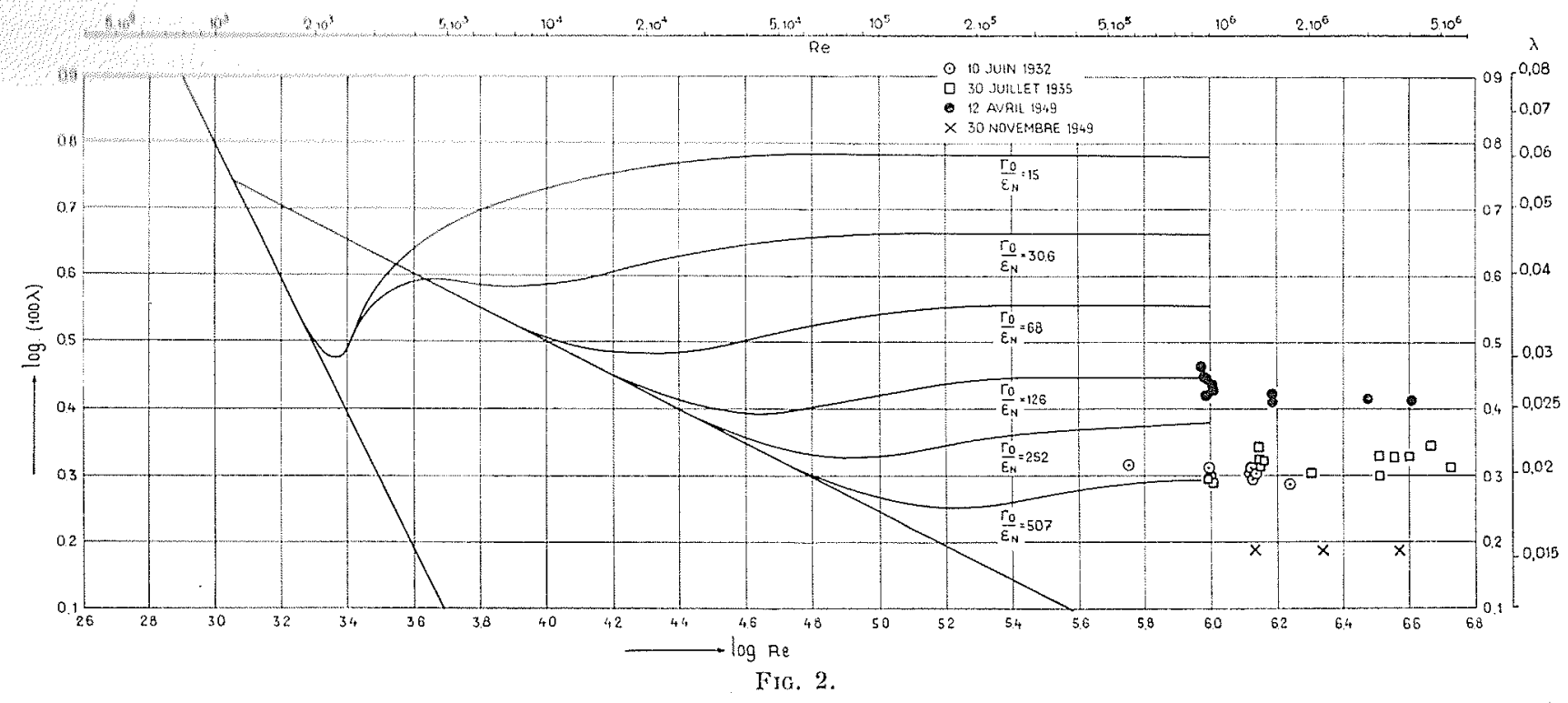

vent être prises égales à celles figurant au tebleau I (1).

(1) Pour les essais du 12 avril 1949, nous n'avons retenu pour le calcul du $\lambda$ moyen, que les 5 valeurs correspondant aux plus faibles valeurs de $\lambda$. Pour les 4 autres essais, effectués en fin de journéc, on n'a pas disposé du temps nécessaire pour l'établissement d'un niveau stable daus la cheminée d'équilibre.
Par la formule classique de Nroundse :

$$
\frac{1}{\sqrt{\lambda}}=1,74+2 \log \frac{r_{0}}{\varepsilon_{X}}
$$

nous en avons déduit les valeurs de $\frac{r_{0}}{\varepsilon_{X}}$ et de là, celles de $\varepsilon_{X}$, rugosité selon Nikuradse. 
Il laul remarquer que l'essai de novembre 1949 correspond à une valeur de $\frac{r_{0}}{\varepsilon_{\mathrm{X}}}$ (1445) bien supérieure à la plus élevée de celles adoptées par Nikuradse: (507). L'extrapolation est done assez. forte.

\section{Tableau I}

\begin{tabular}{|c|c|c|c|}
\hline DATES & $\lambda$ & $\frac{r_{01}}{\varepsilon_{X}}$ & $\varepsilon_{\mathrm{N}} \mathrm{mm}$ \\
\hline 10 juin 1932. & 0,0202 & 452 & 2,15 \\
\hline 30 juillet 1935. & 0,0208 & 398 & 2,45 \\
\hline 12 avril 1949. & 0,0263 & 166,5 & 5,9 \\
\hline 30 novembre 1949. & 0,0154 & 1.445 & 0,675 \\
\hline
\end{tabular}

La rugosité absolue selon Nruurdose déduite de la valeur de $\lambda(5,9 \mathrm{~mm})$ pour le canal recouvert de boue est done nettement supérieure à la rugosité moyenne appréciée par les ouvriers ayant procédé au nettoyage $( \pm 3,5 \mathrm{~mm})$. Leur rapport est 1,65 à 1,70 .

Une correction doit pourtant être apportée à la valeur de $\lambda$ calculée ici comme moyenne pour toute la tuyauterie en ce qui concerne les mesures de novombre 1949.

Comme nous l'avons dit plus haut, tout le canal d'amenée n'a pas été nettoyé, mais seulement les parties bétonnées et nues du tunnel (soit $4.398 \mathrm{~m}$ ), plus le petit tronçon métallique d'Angister (soit $84 \mathrm{~m}$ ), c'est-à-dire en tout $4.482 \mathrm{~m}$ sur une longueur totale de $5.465 \mathrm{~m}$. D'autre part, il a été noté que la "boue déposée sur la conduite métallique ne présente pas l'aspect ondulé caractéristique que l'on trouvait dans le tunnel avant nettoyage $\gg$. Enfin, le nettoyage du tronçon irrégulier de $426 \mathrm{~m}$ ne doit pas avoir eu grand effet, les irrégularités de paroi de plusieurs centimètres étant considérables par rapport à celles du dépôt.

Si l'on admet que les $983 \mathrm{~m}$ non nettoyés avaient un coefficient $\lambda$ compris entre les valeurs moyennes de 1935: 0,0208 ef d'avril 1949: 0,0263 , soit en moyenne 0,0235 , la valeur moyenne du $\lambda$ de la partie nettoyée aurait été, en avril 1949:0,027, peu différente de la valeur calculée plus haut, et en novembre 1949: 0,0135, soit la moitié de la valeur avant nettoyage.

A une valeur de $\lambda$ de 0,027 correspondent des valeurs de $\frac{r_{0}}{\varepsilon_{\mathrm{N}}}$ de 151 et de $\varepsilon_{\mathrm{X}}=6,5 \mathrm{~mm}$ (seIon Nikuradse).

Le rapport entre la valeur absolue de la ru- gosite de Nukunase et la rugosite reelle est $\frac{6,5}{3,5}=1,85$.

Dans un mémoire publié dans la Zeitschrift des Vereins deutscher Ingenieure (1), MM. SExFERTH et KrUgER avaient signalé l'augmentation considérable de $\lambda$ subie, en peu d'années, par la conduite en acier de l'Ecker. Cette r'ésistance, mesurée sur un troncon long de $178,1 \mathrm{~m}$ et d'un diamètre de $496 \mathrm{~mm}$, correspond aux valeurs suivantes de $\lambda$ (variable avec le nombre de REYNOLDS) :

$\lambda=$ en moyenne 0,016 à la mise en marche (1943?) et après nettoyage en juillet 1949 ;

$\lambda=$ en moyenne $0,048(0,0564$ à 0,0412$)$ en f́évrier-mars 1948.

En quelques années, la conduite s'était recouverte d'un dépôt de boue, se présentant sous forme d'ondulations, normales au sens de l'écoulement, hautes d'environ $0,7 \mathrm{~mm}$ et écartées d'environ 3 à $8 \mathrm{~mm}$. Il y correspond une rugosité de NiKuradse de $6,2 \mathrm{~mm}$ (pour $\mathrm{Re}=4,92 \cdot 10^{\circ}$ ) à $14,6 \mathrm{~mm}$ (pour Re $=1,38 \cdot 10^{5}$ ).

SeIFerTh et Kruger avaient pu expliquer la forte proportion entre la rugosité de Nururadse et la rugosité réelle, en recourant à des essais de Schurating (2). Celui-ci a expérimenté une série de types de rugosités, différents du type clas-

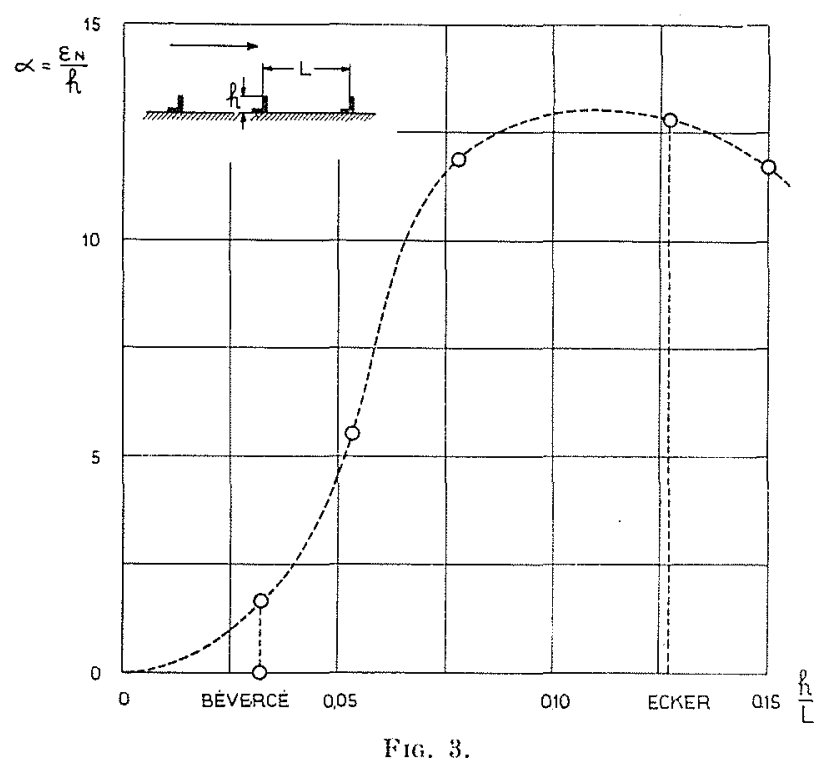

(1) Ueberraschend hohe Reibungsziffer einer Fernumsserleitung (Z.V.d.1.. 11 mars 1950).

(2) Schuchtsa. - Experimentelle Untersuchungen zum Ranhigkeitsproblem (Ingenieur-Archiv, yol. 7, n" 1 , $19366^{\circ}$. 
sique de Nikurabse, parmi lesquels figure celui qu'il appelle «Lange Winkel » : il est constitué de longues cornières disposées sur un fond poli et orientées perpendiculairement à l'écoulement (fig. 3 ).

Trois modèles ont été expérimentés se distinguant par les rapports $\frac{h}{\mathrm{~L}}$ de la hauteur $h$ de la saillie à la distance $\mathrm{L}$ de deux saillies voisines (tableau II). Schucicring a déterminé pour chacun de ces trois modèles le rapport $a=\frac{\varepsilon_{V}}{h}$ de la rugosité équivalente de Nikuradse $\varepsilon_{x}$, à la hauteur h de la saillie.

Tableau II

\begin{tabular}{|c|c|c|c|c|c|}
\hline Montele & L. $\mathrm{mm}$ & h $\mathrm{inm}$ & $\frac{h}{\text { L }}$ & $\varepsilon_{\mathrm{N}} \mathrm{nm}$ & $\alpha$ \\
\hline 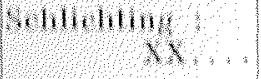 & 60 & 3,2 & 0,0635 & 18,1 & 5,61 \\
\hline XI. & 40 & 3,1 & 0,0775 & 37,0 & 11,9 \\
\hline XII. & 20 & 3 & 0,15 & 35,6 & 11,75 \\
\hline
\end{tabular}

Nous avons tracé au mieux le diagramme de $x$ en fonction de $\frac{h}{\mathrm{~L}}$ passant par ces trois points et aboutissant, tangentiellement, à l'axe des abseisses, à $\alpha=0$ et $\frac{h}{\mathrm{~L}}=0$ (surface polie) (fig. 3 ). Nous y avons fait figurer le point correspondant à la conduite de l'Ecker $\left(\frac{h}{\mathrm{~L}}=\mathrm{env} \cdot \frac{0,7}{5,5}=0,127\right)$ et donnant $\%=12,8$. Avec $\varepsilon_{N}=6,2$ à $14,6 \mathrm{~mm}$, soit en moyenne $10,4 \mathrm{~mm}$, on trouve comme hatuteur moyenne d'irrégularité $\frac{10,4}{12,8}=0,8 \mathrm{~mm}$ très proche de la valcur 0,7 observée.

Le dépôt de boue de Bévereé donnait une valleur moyenne de $\frac{h}{\mathrm{~L}}=\frac{3,5}{110}=0,032$, soit au diagramme $\alpha=1,9$, valeur pratiquement égale à celle constatée plus haut : 1,85 .

Nons nous rendons sans doute bien compte de ce que l'explication que nous proposons a de fragile el de ce qu'il y a d'imprécis dans les bases sur lesquelles elle repose.

Nous pensons cependant pouroir l'énoncer, avec l'espoir que de nouvelles observations, aussi précises que possible, permettront soit de la confirmer, soit de linfirmer.

\section{DIS CUSSION}

M. Ie Président remereie M. Schua davoir bien voulu venir spécialement à paris pour présenter ee mémoire, souligne la clarté de son exposé, et le paradoxe qu'il contient, a savoir la moindre résistance de la conduite vieille après nettoyage vis-á-vis de la conduite neuve.

M. Schlag observe toutefois que la conduite, mise en service en 1929 n'était plus tout à fait neuve lors des premières mesures en 1932 et 1935 ( $^{*}$ ).

En cas de publication. M. le Président suggèe de graduer directement les axes du diagramme en valeurs $\lambda \mathrm{el}$ ik correspondant à l'échelle logarithmique adoptéc.

M. Róménenas rend compte de phénomènes analogues constatés dans une galerie en béton lisse, de diamètre utile comparable a colle de Beveré, alimentant l'usine d'Electricite de France at Nonceau-la-Virolle: une diminution de la puissance maximum constatée peu de temps après la mise en service suscita des mesures de pertes de charge, qui révélèent une augmentation de la

(*) Les premieres années ont pu être assez " lourdes ", le terrin n'étant pas encore stabilise. Ce fait scmble confirme par les puissances naxima atteintes, pour une cote de 495 a 495,50 an réservoir, soit :

$9.900 \mathrm{~kW}$ à $10.000 \mathrm{~kW}$ (en 1930 :

Maximum de $1 / 4$ d'heupe, les 19 et 20 111ai.... 10.100

$9.300 \mathrm{~kW}$ en 1931 :

Maximum de $1 / 4$ a'heure, le 16 mat.......... 9.500

$9.100 \mathrm{~kW}$ en 1932 .

Maximum de $1 / 4$ d'heure, le $1 \mathrm{i}$ novembre...... 9.400 résistance de $53 \%$ en quelques mois; cette perte de charge attribué par M. Róméntéras à un « enduit bioiogique $\gg$. disparut effectivement après grattage de l'enduit et lavage de la galerie, mais reparût bicntôt, au fur et à mesure de la croissance des algues microscopiques poussant sur le béton. M. RÉMÉniéras donne les chiffres des divers essais de Nonceau-la-Virolle, et en tire la valeur de la rugosité $\varepsilon$ de Nikuradse : celle-ci passe de $0,7 \mathrm{~mm}$ quand la galerie est propre à $1,7 \mathrm{~mm}$ quand les algues ont proliféré; ha diffèrence, soit $1 \mathrm{~mm}$, pouvant ctre attribuée aux algues en vie dont les poils assez longs donneraient des pertes de charge plus grandes que celles qui pourraient ètre déduites de leurs dimensions purement géométriques. La suppression des enduits biologiques par l'emploi de peintures stérilisantes se heurte à des conditions de non-toxicité pour les poissons et pour les usagers de l'eau à l'aval. L'intérêt pratique de ces recherches se chiffre annuellement par des millions de kilowatts-heure et paye très largement le lavage des conduites pour lequel on pourrait d'ailleurs trouver des procédés plus rationnels que le travail manuel.

M. Laronde confirme que l'enduit biologique est formé d'algues dont les poils rabattus quand la conduite est vide, lui donnent une apparence lisse, mais penvent flotter dans l'ea et créer à la faveur de la tubblence, une ondulation de surface de l'enveloppe des cxtrémités des algues qui arriverait a produire un effet analogue à celui qu'on constate par les ondulations fixes. Une étude 
complémontaire d'une plaque mise artificiellement dans la condute et tramsporté ensuite en canal vilé pourait permette de préciser l'évolution de ce phénomene an fonction de la vitesse de l'eau.

M. Christian Brav s'est inquieté avec ses collaborateurs a l'Energie des Mers, de la perte de charge et mene de lobstruetion qui potratient ctre causces dans les longs tuyaux de prise d'eau des centrates thermiques en exploilation par les dépots d'organismes sous-malins : algues, coquillages, de... Les biologistes qui ont déjà etudié la question a propos des câbles sous-marins, croient savoir qu'à l'abri de la lumiere (dans les grandes profondent's sous-marines ou les conduites opaques) seules les especes animales peuvent proliférer, ce qui semble en contradiction ave les constatalions faites par les hydrat liciens dans les conduites forcés ou galeries. Cependant, les thermodynamiciens, qui ont eprouve de semblables difficultés pour leurs prises d'eau en Scinc, ont detruit. les micro-organismes par injection de chlore dans les puisards.

M. Gace confine lemploi du chlore dans les entrales lhermiques parisiennes et précise qu'il est injecté sporadiquement pendant une demi-heure loutes les huit heures; au bord de la mer dans certaines centrales thermiques, on tue les moules incrustées dans les conduites d'amené par choc thermique, en inversant les cireulat tions deau clatude et d'eat froide.

M. Scanatio prése que laugmentation de résistane de ia conduite de Beveré contait environ $640.000 \mathrm{k}$ Wh par an, soit, en valeur, la moilie du prix du neltoyage.

M. Le President conclut, d'apres les commentaires, qu'il y aula probablement encore quelques experiences a fare pour préciser la nature de « l'enduit biologicue $»$ son influenee sur les pertes de charge et les moyens de l'éliminer.

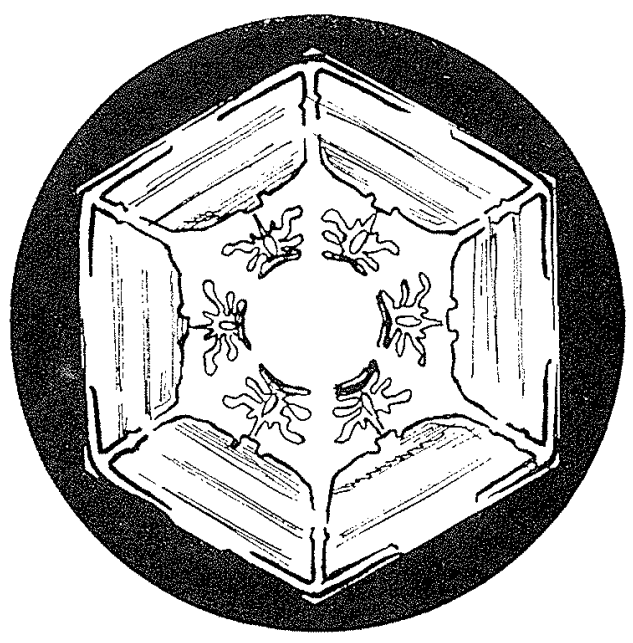

